\title{
Fault Tolerance Analysis of Modified Irregular Augmented Shuffle Exchange Network (MIASEN)
}

\author{
Shobha Arya*, Nipur Singh** \\ *(Department of Computer Science, Gurukul Kangri Vishwavidyalaya, India, \\ Email:01shobha@gmail.com) \\ **(Department of Computer Science, Gurukul Kangri Vishwavidyalaya, India, \\ Email:nipur@gkv.ac.in) \\ Corresponding author: Shobha Arya
}

\begin{abstract}
Multistage Interconnection Networks (MINs) play a vivacious position to perform high performance within the discipline of VLSI, broadband communications, parallel and distributed systems designs. The problem of fault tolerance and cost effectiveness are the predominant challenges for calculating the overall performance of MINs. A MIN is better fault tolerant, if it may deal with the extra faults come across in different stages. The fault tolerance of proposed Modified Irregular Augmented Shuffle Exchange Network (MIASEN) [13] is examined in terms of bandwidth, processor utilization, throughput, probability of acceptance, and processing power. The performance and evaluation analysis indicates that the MIASEN is more fault tolerant than the existing Modified Alpha Network (MALN).
\end{abstract}

Keywords - Fault tolerance, Multistage Interconnection Network

\section{INTRODUCTION}

The performance of high-performance computing systems relies heavily on the efficiency of the multistage interconnection networks (MINs). Because of its high speed and low cost, MINs become more popular for multiprocessor systems as compared to single-processor systems. The problem of lack of fault tolerance is the major issue in MINs. The performance of MINs is depending on fault tolerance capability of that network. For example, single path MINs are less fault tolerant as compared to multipath MINs. A MIN is less reliable if it tolerate minimum faults. Fault tolerance can be increased by following methods:

(i) To increase the number of stages

(ii) To increase the number of links

(iii) To increase the number of switches

(iv) To replicate the entire network

In recent years, various research works have been done on fault tolerance techniques and to design new MIN to increase the fault-tolerance [16]. In this research paper, the fault tolerance analysis and routing algorithm of proposed Modified Irregular Augmented Shuffle Exchange Network (MIASEN) [13] have been discussed. The fault tolerance of proposed Modified Irregular Augmented Shuffle Exchange Network (MIASEN)
[13] is compared with existing Modified Alfa Networks (MALN) [1].

Section 2 depicts basic structure of Modified Alfa Networks (MALN) [1] and Modified Irregular Augmented Shuffle Exchange Network (MIASEN) [13]. The routing algorithm of MIASEN is also discussed in Section 3. Section 4 focuses on the Fault-tolerance analysis of MIASEN on different parameters. Section 5 concentrates on the results and comparison analysis of MINs. In Section 6, the conclusion has been presented.

\section{STRUCTURE OF MULTISTAGE INTERCONNECTION NETWORKS}

In this paper, the focus is on fault tolerance of irregular MINs. The basic structure of Modified Alfa Networks (MALN) [1] and proposed Modified Irregular Augmented Shuffle Exchange Network (MIASEN) [13] are discussed below.

\subsection{Modified Alpha Network}

Modified Alfa Networks (MALN) [1] consists of $\mathrm{N}$ number of source and destination addresses with $(2 \mathrm{~m}-2)$ stages where $m=\log _{2}(\mathrm{~N} / 2)$. Each source is linked with $2 \times 1$ MUX and each destination is linked with a $1 \times 2$ demultiplexer. All stages except the last stage consist of switching elements (SE) of 
size $3 \times 3$ and the last stage consists of switching elements of size $2 \times 2$. The switching elements in the stages n-3, n-2 and n-1 are connected to each other through auxiliary or alternate links where $n=\log _{2} \mathrm{~N}$. The network is divided into two identical subnetworks G0 and G1 [1].

\subsection{Modified Irregular Augmented Shuffle Exchange Network}

The Modified Irregular Augmented Shuffle Exchange Network (MIASEN) [13] consists of N number of source and $\mathrm{N}$ number of destination addresses. The MIASEN has $\mathrm{N} \times \mathrm{N}$ network size with $\left[\left(\log _{2} \mathrm{~N}\right)-1\right]$ number of stages. The first and last stages have N/2 switches (SEs). The size of each SE of first stage is $3 \times 3$ and the size of each SE of last stage is $2 \times 2$. The middle stage has (N/8) number of SEs and size of each SE is $5 \times 5$. MIASEN has $\mathrm{N}$ number of multiplexers (MUX) with size $2 \times 1$ each and $\mathrm{N}$ number of demultiplexers (DEMUX) with size $1 \times 2$ each. Each $\mathrm{SE}$ of the first stage and each SE of the last stage connected with two MUX and two DEMUX respectively. A $16 \times 16$ network size MIASEN with 3 numbers of stages is shown in Fig. 1.

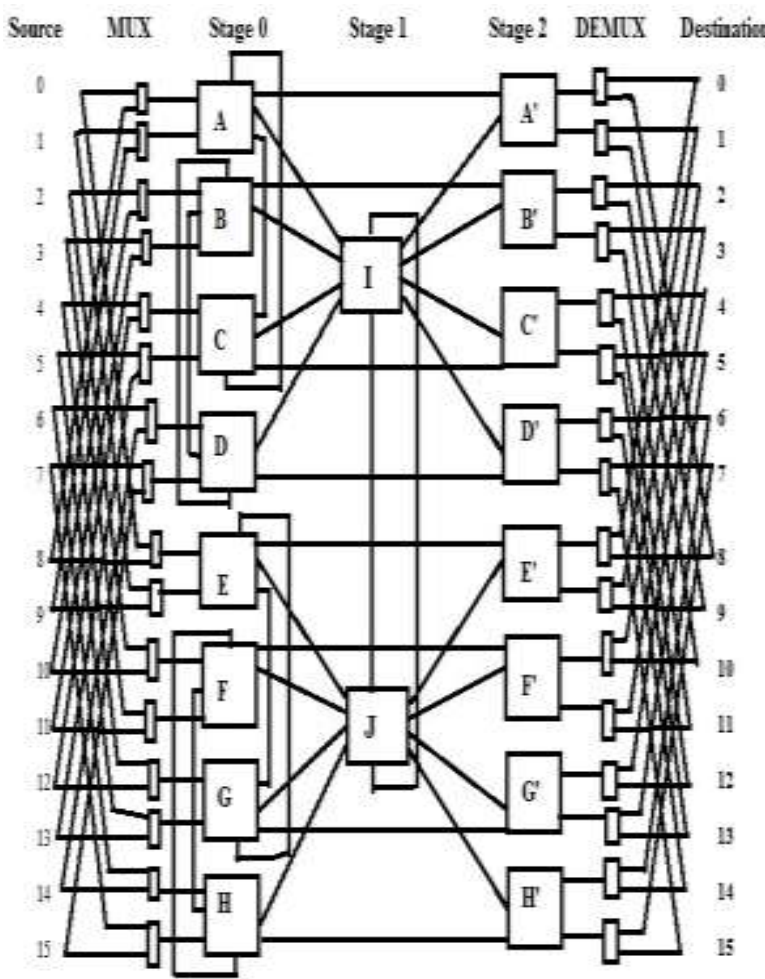

Fig. 1 Modified Irregular Augmented Shuffle Exchange Network (MIASEN)

MIASEN is fault tolerant network and reliable MIN if any failure occurs in any switch in the network then there will be an alternate path to work properly. Here, Table 1 displays the symbols and their meaning, which are used in this paper.
Table I. Symbol Table of SE

\begin{tabular}{|l|l|}
\hline Symbol & \multicolumn{1}{|c|}{ Meaning } \\
\hline $\mathrm{PSE}_{1}$ & Primary Switching Element of stage First \\
\hline $\mathrm{ASE}_{1}$ & Auxiliary Switching Element of stage First \\
\hline $\mathrm{PSE}_{2}$ & Primary Switching Element of stage Second \\
\hline $\mathrm{ASE}_{2}$ & Auxiliary Switching Element of stage Second \\
\hline $\mathrm{PSE}_{3}$ & Primary Switching Element of stage Third \\
\hline $\mathrm{ASE}_{3}$ & Auxiliary Switching Element of stage Third \\
\hline
\end{tabular}

\section{ROUTING ALGORITHM OF MIASEN}

In MIASEN, if a switching element (SE) is directly associated with any source or any destination, then it is called primary switching element (SE) of that particular source or destination address. If a switching element (SE) is indirectly associated with any source or any destination via auxiliary or alternate links, then it is called first or second alternate SE. For example, A is the primary $\mathrm{SE}\left(\mathrm{PSE}_{1}\right)$ and $\mathrm{E}$ is secondary $\mathrm{SE}\left(\mathrm{ASE}_{1}\right)$ for source 0 . In routing of MIASEN, first step is to obtain the source address and its corresponding destination address. If there is no fault in primary SE $\left(\mathrm{PSE}_{1}\right)$ of first stage, then send the request to its primary switching element $\left(\mathrm{PSE}_{1}\right)$. If there is any fault in PSE1 then packet is rerouted and send via auxiliary or alternate switching element $\left(\mathrm{ASE}_{1}\right)$ of stage first. The same logic is used for SEs of second and third stage. If there is more than one SE is faulty then request will be dropped otherwise send packet to its appropriate node or destination. Hence, MIASEN is a single switch fault tolerant MIN.

\section{BEGIN}

if $\mathrm{PSE}_{1}==\mathrm{FB} / / \mathrm{FB}$ means busy or faulty

then $\mathrm{ASE}_{1}$

else Send Request to Next Appropriate Node

// Here Node may be SE of next stage

if $\mathrm{ASE}_{1}==\mathrm{FB}$

then Drop the Request

if $\mathrm{PSE}_{2}==\mathrm{FB}$

then $\mathrm{ASE}_{2}$ 
else Send Request to Appropriate SE of Third Stage

if $\mathrm{ASE}_{2}==\mathrm{FB}$

then Drop the Request

else Send Request to Appropriate SE of Third Stage

$$
\text { if } \mathrm{PSE}_{3}==\mathrm{FB}
$$

then $\mathrm{ASE}_{3}$ Node

else Send Request to Appropriate Destination

$$
\text { if } \mathrm{ASE}_{3}==\mathrm{FB}
$$

then Drop the Request

End

\section{FAULT TOLERANCE ANALYSIS OF MIASEN AND MALN}

The fault tolerance analysis of MIASEN and MALN is measured in terms of performance evaluation parameters such as probability of acceptance, bandwidth, throughput, processor utilization, processing power.

\subsection{Request Generation Probability (p)}

"The expected numbers of destination receiving request in a given cycle is known as bandwidth of network [3][14]". Let the network size is $\mathrm{N} \times \mathrm{N}$. Then the bandwidth will be as follows:

$$
\mathrm{BW}=\mathrm{N} \times \mathrm{p}_{\mathrm{n}}
$$

Probability equation for MALN [1]:

$$
\mathrm{p}_{0}=\mathrm{p}
$$

Request generation probability for first stage

$$
\mathrm{p}_{1}=1-\left(1-\mathrm{p}_{0} / 3\right)^{3}
$$

Request generation probability for second stage

$$
\mathrm{p}_{2}=1-\left(1-\mathrm{p}_{1} / 6\right)^{3}
$$

Request generation probability for third stage

$$
p_{3}=1-\left(1-p_{2} / 3\right)^{3}
$$

Request generation probability for fourth stage

$$
\mathrm{p}_{4}=1-\left\{\left(1-\mathrm{p}_{3}\right) \times\left(1-\mathrm{p}_{1} / 2\right)\right\}^{2}
$$

Probability equation for MIASEN:

$$
\mathrm{p}_{0}=\mathrm{p}
$$

Request generation probability for first stage

$$
\mathrm{p}_{1}=1-\left(1-\mathrm{p}_{0} / 3\right)^{3}
$$

Request generation probability for second stage

$$
\mathrm{p}_{2}=1-\left(1-\mathrm{p}_{1} / 5\right)^{5}
$$

Request generation probability for third stage

$$
\mathrm{p}_{3}=1-\left[\left(1-\mathrm{p}_{2}\right) *\left(1-\mathrm{p}_{1} / 2\right)\right]^{2}
$$

\subsection{Data Transmission Time}

"It is time that all generated data packets take from source to the given number of destinations [14]."

If network is non-faulty, then it is given as follows:

$\mathrm{t}=\left(\mathrm{N}_{\mathrm{n}}-1\right) \times \mathrm{T} \times \mathrm{N}_{\mathrm{dp}} \times \mathrm{D}_{\mathrm{n}}$

If network has single switch fault, then it is given as follows:

$\mathrm{t}_{\mathrm{SF}}=\mathrm{t}+(\mathrm{S} \times \mathrm{T})$

Where,

$\mathrm{N}_{\mathrm{n}}=$ Number of nodes including source and destination

$\mathrm{T}=$ Routing Time between two nodes

$\mathrm{D}_{\mathrm{n}}=$ Number of destinations

$\mathrm{N}_{\mathrm{dp}}=$ Total number of generated data packets on a source node

$\mathrm{S}=$ total number of stages

$\mathrm{t}=$ If network is non-faulty, then data transmission time

$\mathrm{t}_{\mathrm{SF}}=$ If network is single switch faulty, then data transmission time

\subsection{Bandwidth}

"The expected numbers of destination receiving request in a given cycle is known as bandwidth of network [3][14]". Let the network size is $\mathrm{N} \times \mathrm{N}$.

Then the bandwidth will be as follows:

$$
\mathrm{BW}=\mathrm{N} \times \mathrm{p}_{\mathrm{n}}
$$

\subsection{Probability of Acceptance (PA)}

"Probability of Acceptance (PA) is the number of request accepted by the destination side that is sent by the source side in a transfer cycle [3][14]". It is calculated as follows:

$$
\mathrm{PA}=\left[\mathrm{BW} /\left(\mathrm{D}_{\mathrm{n}} \times \mathrm{p}\right)\right]
$$

\subsection{Throughput (TP)}

"It is the maximum number of traffic per unit time accepted by a network is called throughput [3][14]". 


$$
\mathrm{TP}=(\mathrm{BW} / \mathrm{N} \times \mathrm{t})
$$

Where, $\mathrm{t}$ is the data transmission time.

\subsection{Processor Utilization (PU)}

"The percentage of time the processor is active doing computation without retrieving the global memory is known as processor utilization [3][14]".

$$
\mathrm{PU}=(\mathrm{BW} / \mathrm{N} \times \mathrm{p} \times \mathrm{t})
$$

\subsection{Processing Power (PP)}

"The sum of processor utilization over the number of processors is known as processing power [3]".

$$
\mathrm{PP}=(\mathrm{N} \times \mathrm{PU})
$$

\section{COMPARISON AND ANALYSIS}

The performance analysis and comparison is determined for MALN and MIASEN on above parameters in faulty and non-faulty environment. Let the data packet is transferred from source 1 to destination 6 . Let the routing time between two nodes is $0.01 \mathrm{~ms}$, when network is non-faulty and $0.02 \mathrm{~ms}$, when there is a single switch fault in network. Nodes can be anything either source or destination or SE. Let the value of request generation probability or offered load $(\mathrm{p})$ is $0.1,0.2$, $0.3,0.4,0.5,0.6,0.7,0.8,0.9$, and 1 . Therefore, comparison between MALN and MIASEN is performed by bandwidth, probability of acceptance, throughput, processor utilization, and processing power in non-faulty and single switch fault environment.

\subsection{Bandwidth (BW)}

The performance analysis shows that the bandwidth of MIASEN is better than MALN.

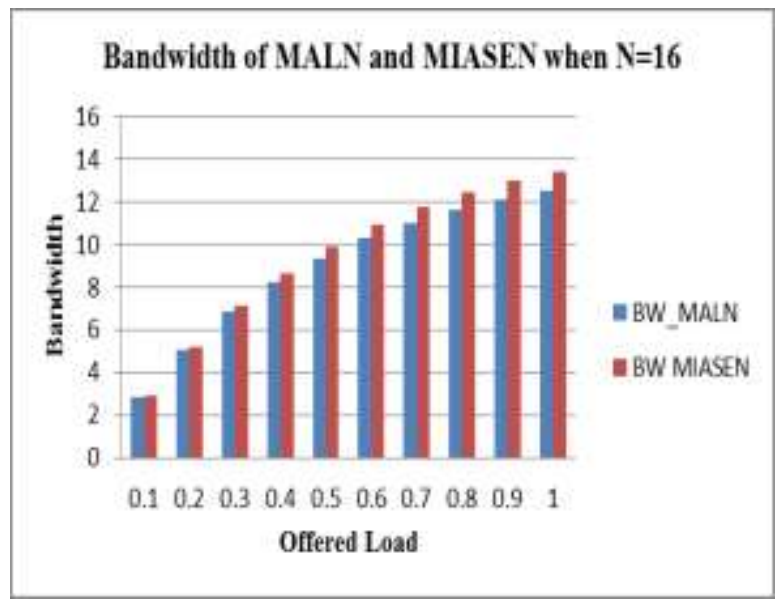

Fig. 2 Bandwidth Comparison under Non-Faulty and Faulty conditions

\subsection{Probability of Acceptance (PA)}

The Probability of Acceptance of MIASEN is greater than MALN.

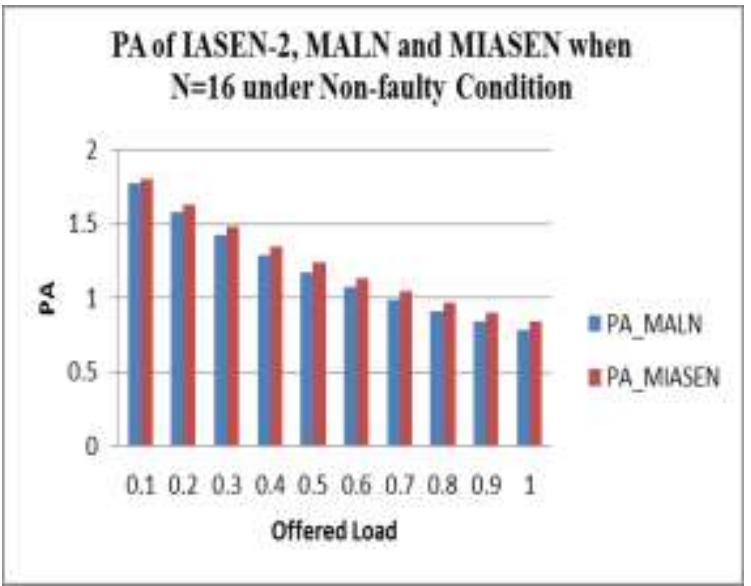

Fig. 3 Probability of Acceptance under Non-Faulty and Faulty conditions

\subsection{Throughput (TP)}

The performance comparison shows that throughput of MIASEN is better than MALN under non-faulty (without fault) and faulty (with single switch fault) conditions. 


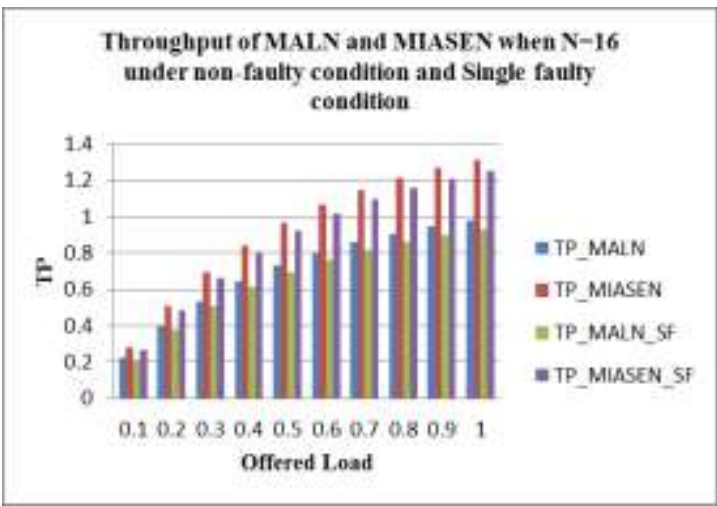

Fig. 4 Throughput under Non-Faulty and Faulty conditions

\subsection{Processor Utilization (PU)}

Processor utilization of MIASEN is better than MALN in both conditions.

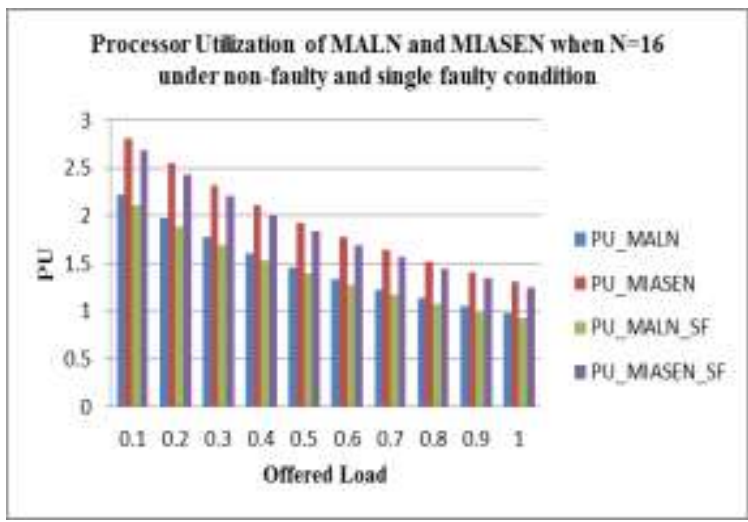

Fig. 5 Processor Utilization under Non-Faulty and Faulty conditions

\subsection{Processing Power (PP)}

Processing Power (PP) of MIASEN is greater than PP of MALN under faulty and non-faulty conditions.

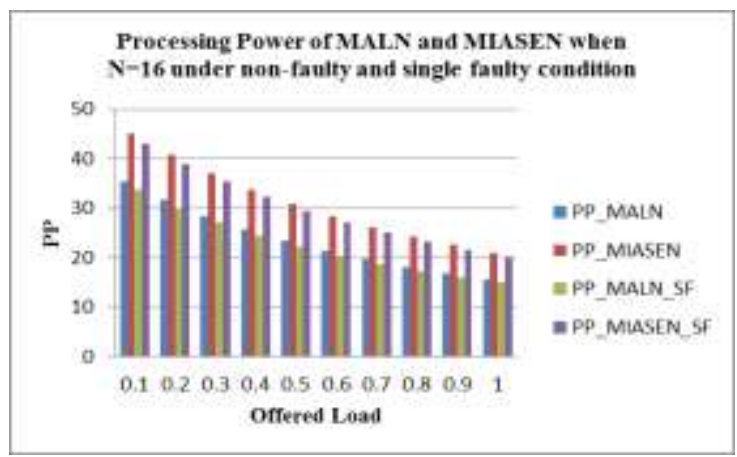

Fig. 6 Processing Power under Non-Faulty and faulty conditions

\section{CONCLUSION}

The results and analysis shows that the performance evaluation parameters of MIASEN give better results than that of MALN. The throughput, bandwidth, probability of acceptance, processor utilization and processing power of MIASEN are greater than MALN. Therefore, we can conclude that the Modified Irregular Augmented Shuffle exchange Network (MIASEN) is more fault-tolerant as compared to MALN.

\section{REFERENCES}

[1] Gupta A. and Bansal P. K., "Fault tolerant irregular modified alpha network and evaluation of performance parameters". International Journal of Computer Applications. vol.4, no. 1, 9-13, 2010.

[2] Rita Mahajan and Renu Vig, "A Performance and reliability analysis of new fault-tolerant advance omega network", WSEAS Transactions on Computers, vol.7, no.8, 12801290, 2008.

[3] Mamta Ghai, Vinay Chopra, and Karamjit Kaur Cheema, "Performance analysis of fault-tolerant irregular baseline multistage interconnection network", International Journal on Computer Science and Engineering, vol. 2, no. 9, pp. 3079-3084, 2010.

[4]C. C. Fan, and J. Bruck, "Tolerating multiple faults in multistage interconnection networks with minimal extra stages", Comput. IEEE Trans., Vol. 49, no. 9, pp. 9981004, 2000.

[5]Y. Yang, and J. Wang,"Routing permutations on baseline networks with node-disjoint paths", Parallel Distribut. Syst. IEEE Trans., Vol. 16, no. 8, pp. 73746, 2005.

[6]P. H. Pham, J. Song, J. Park, and C. Kim, "Design and implementation of an on-chip permutation network for multiprocessor system-on-chip", Very Large Scale Integration (VLSI) Syst. IEEE Trans., Vol. 21, no. 1, pp. 1537, 2013.

[7] J. Nathaniel Davis, William, Tsun-yuk Hsu and H. J. Siegel, "Fault location techniques for distributed control interconnection networks", IEEE Transactions on Computers, Vol. C-34, No.10, October 1985.

[8] V. P. Kumar and S. M. Reddy, "Design and analysis of faulttolerant multistage interconnection networks with low link complexity", Proceedings of $12^{\text {th }}$ International Symposium on Computer Architecture, 1985 June, pp. 376-386.

[9]Bansal, P. K., Joshi, R.C., Singh, K., "On a Fault tolerant Multistage Interconnection Network", International Journal of Electronics and Electrical Engineering, 20(4), pp. 335-345,1994.

[10] Aggarwal R., Aggarwal H. and Kaur L., "On Bandwidth analysis of Irregular Fault-tolerant Multistage Interconnection networks", International Review on Computers and Software, Vol. 3, No. 2, March 2008, pp. 199-202.

[11] Bansal P.K, Singh Kuldeep and Joshi R.C., "Quad Tree: A Cost-Effective Fault-Tolerant Multistage Interconnection network", Proceeding of International Conference IEEE INFOCOM, 1992, pp. 6D.1.1-6D.1.7.

[12] Karamjit Kaur Cheema, Rinkle Aggarwal, "Design Scheme and Performance Evaluation of a new Faulttolerant Multistage Interconnection Network", IJCSNS International Journal of Computer Science and Network Security, VOL.9 No.9, pp. 270-276, September 2009.

[13] Shobha Arya, Nipur Singh, "Reliability Analysis of Modified Irregular Augmented Shuffle Exchange Network (MIASEN)", International Journal of Computer Science Issues, Vol. 14, No. 3, pp. 59-64, May 2017.

[14] Shobha Arya, Nipur Singh, "Performance Analysis of Fault Tolerant Advanced Irregular Shuffle Exchange Network (AISEN)", Proceeding of ICTCS '16, March 04$05,2016$. 